\title{
Introduction to a Special Section on the Effects of the Dating Matters Model on Secondary Outcomes: Results from a Comparative Effectiveness Cluster Randomized Controlled Trial
}

\author{
Phyllis Holditch Niolon ${ }^{1}$
}

Accepted: 31 October 2020 / Published online: 17 November 2020

(C) This is a U.S. government work and not under copyright protection in the U.S.; foreign copyright protection may apply 2020, corrected publication 2021

\begin{abstract}
Teen dating violence (TDV) affects millions of young people in the USA each year (Basile et al. 2020) and is associated with a myriad of negative consequences across the lifespan, including placing individuals at greater risk for experiencing intimate partner violence (IPV) in their more permanent relationships in adulthood (Exner-Cortens et al. Pediatrics 131(1):71-78 ExnerCortens et al. 2013; Exner-Cortens et al. Journal of Adolescent Health 60(2):176-183 Exner-Cortens et al. 2017). The CDC developed the Dating Matters ${ }^{\circledR}$ : Strategies to Promote Healthy Teen Relationships comprehensive prevention model to prevent TDV and its consequences among young people, and it was found to be effective at reducing TDV perpetration and victimization compared with another evidence-based program (Niolon et al. American Journal of Preventive Medicine 57(1):13-23 Niolon et al. 2019). Dating Matters addresses multiple risk and protective factors for TDV through its multiple components, many of which are shared risk and protective factors for other forms of violence and risk behaviors among adolescents. This article introduces this special section, which includes three papers examining these secondary outcomes of the Dating Matters comparative effectiveness, multi-site, longitudinal cluster randomized controlled trial and concludes with an invited commentary by Debnam and Temple (2020). This introduction briefly discusses the Dating Matters comprehensive prevention model, the comparative effectiveness trial used to evaluate effectiveness, the outcomes examined by the three papers included in this special section and the commentary from external reviewers. This special section makes an important contribution to the field of violence prevention, highlighting a preventive intervention for TDV that addresses a constellation of risk and protective factors and demonstrating its effects on multiple adolescent risk and violence outcomes.
\end{abstract}

Keywords Dating matters $\cdot$ Prevention $\cdot$ Randomized controlled trial $(\mathrm{RCT})$

Many in the field of violence prevention are calling for more integrated approaches to violence prevention - strategies that prevent multiple forms of violence simultaneously by addressing shared risk and protective factors for these forms of violence (Centers for Disease Control and Prevention 2016; Niolon et al. in press). Such an integrated approach creates both an efficient use of prevention resources and a lower burden on those responsible for implementing prevention strategies (e.g., schools who may be interested in preventing bullying and peer violence, teen dating violence, and substance abuse, but may not have the capacity to implement three

Phyllis Holditch Niolon pniolon@cdc.gov

1 Division of Violence Prevention, Centers for Disease Control and Prevention, 4770 Buford Highway, MS 106-10, Atlanta, GA 30341 , USA separate prevention programs). Evidence suggests a strong rationale for the effectiveness of prevention strategies aimed at reducing multiple forms of violence (Centers for Disease Control and Prevention 2016).

For example, many forms of violence perpetration share the same risk and protective factors, with many of the strongest occurring early in the lifespan; second, those who perpetrate one form of violence are at higher risk to perpetrate other forms of violence, and similarly, those who are victims of one form of violence are at higher risk to experience other forms of violence; and third, different forms of violence share many common consequences and costs to society (Centers for Disease Control and Prevention 2016). Therefore, it is likely that prevention strategies that attempt to impact the strongest risk and protective factors for multiple forms of violence, especially those that occur early in the lifespan, are likely to reduce perpetration of multiple forms of violence, thereby reducing risk of perpetrating and experiencing other forms 
of violence later in the lifespan. This type of early prevention of multiple forms of interpersonal violence has the potential to reduce the negative and profound impacts that violence can have on individuals, families, communities, and societies. This article provides an introduction to a group of papers reporting secondary outcomes middle school results of the randomized controlled trial of Dating Matters ${ }^{\circledR}$ : Strategies to Promote Healthy Teen Relationships, which is a comprehensive prevention model designed to impact multiple forms of violence and risk factors for violence through addressing a constellation of risk and protective factors for these behaviors. This introduction provides a description of the Dating Matters (DM) comprehensive prevention model, a summary of the comparative effectiveness trial design used to evaluate it, an overview of the primary outcomes, an introduction to the secondary outcomes examined by the three papers included in this special section, and a brief discussion of the commentary from Debnam and Temple (2020).

In 2009, guided by a request from Congress, CDC began work to advance the teen dating violence (TDV) prevention field. TDV is a type of intimate partner violence that occurs in teen or adolescent relationships and can include physical violence, sexual violence, psychological aggression, and stalking (Centers for Disease Control and Prevention 2020). It affects millions of young people in the USA each year; a recent study found that 1 in 8 high school students in the USA report experiencing either physical and/or sexual TDV in the last year (Basile et al. 2020). TDV is associated with a myriad of negative consequences across the lifespan, including depression, antisocial behavior, substance use, suicidal ideation, and placing individuals at greater risk for experiencing intimate partner violence (IPV) in their more permanent relationships in adulthood (Exner-Cortens et al. 2013; Exner-Cortens et al. 2017). CDC identified several existing gaps in prevention of TDV and developed a comprehensive prevention model and implementation plan to address these gaps (Tharp et al. 2011; Niolon et al. 2019). At the time that Dating Matters was being developed, a few interventions that were effective at reducing TDV existed, but they were single-component, individuallevel preventive interventions implemented in school settings. In other areas of violence prevention, however, it was clear that multi-component interventions implemented at multiple levels of the social ecology (e.g., individual, relational, community, societal) were more successful at enhancing population-wide benefits and sustainability than singlecomponent interventions (David-Ferdon and Simon 2014). Additionally, national estimates indicated that about 1 in 10 high school students was experiencing TDV, a statistic which had remained stable for the preceding decade (Eaton et al. 2010); therefore, it was clear that to maximize primary prevention of teen dating violence, efforts are needed with young people before they reach high school. Finally, although empirical data on risk factors for teen dating violence suggested that youth in high risk, urban communities might be at higher risk for experiencing and perpetrating teen dating violence (Reed et al. 2011; Walton et al. 2009), no interventions to date had been tested in these communities. Therefore, CDC developed a comprehensive prevention model to address risk and protective factors for teen dating violence at multiple levels of the social ecology, to be implemented in middle schools and with communities in high risk urban areas.

During 2 years of formative research and planning, the Dating Matters comprehensive prevention model was designed and developed by CDC. The goal of Dating Matters was to create a comprehensive, multi-component model that was built on the existing evidence-base; therefore some of the components were existing evidence-based interventions, while other components were developed by $\mathrm{CDC}$, using the themes and content of the evidence-based components as a guide. The Dating Matters comprehensive prevention model consists of the following components along the levels of the social ecological model: (1) at the individual level, the youth programs consist of in-school programs for students in 6th grade (CDC developed), 7th grade (CDC developed), and 8th grade (the evidence-based Safe Dates curriculum; Foshee et al. 2004); (2) at the relationship level, the parent programs were offered to parents of 6th graders (the adapted, evidence-based program Parents Matter! for Dating Matters; Forehand et al. 2007), parents of 7th graders (CDC developed), and parents of 8th graders (Families for Safe Dates; Foshee et al. 2012); (3) at the relationship/community level, $\mathrm{CDC}$ developed the online educator training for teachers and staff within a school, which provided information and resources on teen dating violence and motivated participants to engage in prevention efforts in their schools; 4) at the relationship/community level, the CDCdeveloped youth communications program (i2i: What $R U$ Looking 4) was led by near-peer brand ambassadors (highschool aged youth from the same communities) and reinforced messages about healthy relationships through community events and activities and the provision of print and online resources; and 5) at the community level, CDC developed resources and materials and provided support to local health departments and community partners on building capacity to implement TDV prevention strategies (Dating Matters Capacity and Planning Tool); on using health indicator data to assess and monitor progress; and on assessing and implementing policies that impact TDV (Interactive Guide to Informing Policy). The multiple components of the Dating Matters comprehensive prevention model and their corresponding levels of the social ecology are depicted in Fig. 1.

After 2 years of planning and intervention design, CDC launched a demonstration project for the implementation of Dating Matters, and a CDC-led, multi-site cluster randomized controlled trial to evaluate the demonstration project. The demonstration project awarded non-research cooperative agreements to four local health departments in US cities: 
Fig. 1 The Dating Matters ${ }^{\circledR}$ Comprehensive Teen Dating Violence Prevention Model Learn more: https://www.cdc. gov/violenceprevention/ intimatepartnerviolence/ datingmatters/

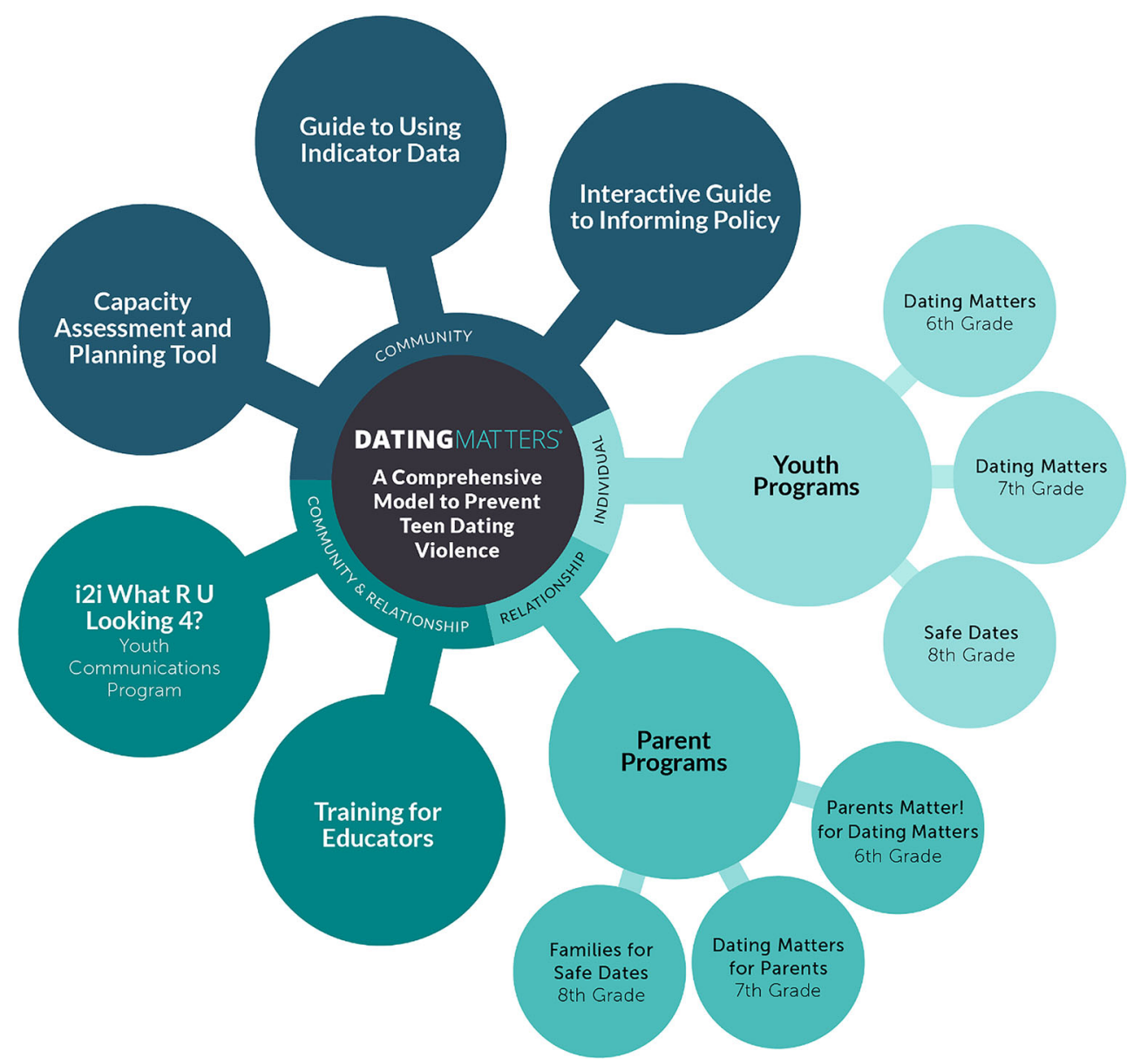

Alameda County (Oakland), CA; Baltimore, MD; Broward County (Ft. Lauderdale), FL; and Chicago, IL. Specifically, recipient health departments based in metropolitan statistical areas of 1 million or more were eligible to apply and had to identify 10-12 neighborhood middle schools in high-risk urban areas (high risk was defined for the purpose of this project as above average rates of poverty and crime) that would implement one of the two models of TDV prevention. The funding announcement explained that the CDC would conduct the random assignment of schools to one of the two models of prevention and that they would participate in a cross-site evaluation; each site was funded for 5 years, with one planning year and 4 years of implementation. The health departments collaborated with $\mathrm{CDC}$ and the evaluation contractor to ensure the integrity of the evaluation (see Niolon et al. 2016 for more details).

The effectiveness trial was conducted using a comparative effectiveness, multi-site, longitudinal, cluster randomized controlled trial design and was funded through a series of two contracts. Within each site, we randomly assigned half of the schools to implement the Dating Matters (DM) comprehensive model and half of the schools to implement our standard of care (SoC) comparison, which was the evidencebased teen dating violence prevention program, Safe Dates
(Foshee et al. 2004). At the time that Dating Matters was being developed, Safe Dates was one of two evidence-based TDV prevention programs, was the only one that demonstrated effects on both perpetration and victimization, had primary and secondary prevention effects, and was effective for both boys and girls; consequently, it was chosen as one of the evidencebased programs incorporated into the Dating Matters model, as well as the standard of care comparison for the evaluation.

This comparative effectiveness approach was selected for practical reasons; evidence-based programs already existed for TDV prevention, and Safe Dates was at the time the most widely implemented TDV prevention program in the USA. Therefore, CDC decided to test whether the Dating Matters comprehensive model was significantly more effective than what was already available to communities in preventing TDV and other adolescent risk outcomes (Niolon et al. 2016). The first evaluation contract overlapped with the implementation non-research cooperative agreements in that it had a year of planning with the sites, and 4 years of data collection, coinciding with 4 years of implementation. After obtaining parental consent and student assent, students were surveyed at school using paper and pencil administration in the fall and spring during each year of implementation. Over the four years of implementation, five cohorts of students were 
surveyed: students in 6th, 7th, and 8th graders in the first year of implementation (Cohorts 3, 2, and 1, respectively), and 6th graders within the second and third years of implementation (Cohorts 4 and 5, respectively). A second evaluation contract allowed us to follow our sample into high school; students were assessed once per year in the spring of 9th-11th grades. More details on the evaluation design can be found elsewhere (Niolon et al. 2016; Niolon et al. 2019).

The initial outcome analyses examined primary and secondary outcomes across the six time points in middle school among the two cohorts (grades) of students who had the opportunity to be exposed to all three middle school grades of the Dating Matters components if they were in the DM condition (cohorts 3 and 4, 6th graders in the first and second years of implementation). After imputing missing data (details available in Niolon et al. 2019), hypotheses were examined using multiple group structural equation models. DM students were compared with SoC students among 4 groups divided by biological sex and cohort (Cohort 3 females, Cohort 3 males, Cohort 4 females, Cohort 4 males).

For the study's primary outcomes, it was hypothesized that students in the DM schools would report less perpetration and victimization of dating violence, less use of negative conflict resolution styles with dating partners, and greater use of positive relationship behaviors with dating partners across the 3 years of middle school when compared with students in the SoC condition. As hypothesized, across most time points and in most groups, students in DM schools reported less perpetration of TDV, less victimization of TDV, and less use of negative conflict resolution strategies with their dating partners. However, all students reported high use of positive relationship behaviors, and no differences emerged between groups (Niolon et al. 2019). Due to the themes and content addressed across the Dating Matters comprehensive prevention model, we also expected that DM students would report less engagement in/exposure to several secondary outcomes as well. The Dating Matters comprehensive model addressed many social-emotional learning principles, such as empathy, respect for oneself and for others, understanding and respecting boundaries, as well as teaching a number of skills related to all interpersonal relationships, such as conflict management, communication skills, and emotion regulation. Therefore, we hypothesized that DM students would also report lower rates of interpersonal violence outside of dating relationships and lower rates of substance use and other delinquent behaviors than SoC students. The papers in this special section report findings on three sets of secondary outcomes: the Vivolo-Kantor et al. paper examines outcomes related to bullying and peer violence; specifically this paper examines the differences between students in DM and SoC schools on perpetration and victimization of physical violence, bullying, and cyberbullying; the DeGue et al. paper examines differences on perpetration and victimization of sexual violence and sexual harassment; and the Estefan et al. paper examines differences on weapon carrying, alcohol and substance use, and other delinquent behaviors (e.g., damaging property, stealing, selling drugs, etc.). Although TDV and relationship behaviors were the primary outcomes targeted by the intervention, the Dating Matters comprehensive model addressed a number of shared risk and protective factors for multiple forms of violence, and it is worthwhile to examine the effects of the intervention on these secondary outcomes as well (Centers for Disease Control and Prevention 2016).

The commentary by Debnam and Temple (2020) identifies a number of strengths and weaknesses of the Dating Matters comprehensive prevention model. One of the most notable points they raise is that the fact that the model has multiple components across the various levels of the social ecology is both a major strength and a significant weakness. While the authors note that the comprehensive nature of this multi-component model is likely what contributes to its success on the variety of outcomes examined in this special section when compared with the school-based curriculum SoC model, they point out, very astutely, that these multiple components may also make it very difficult and costly for resource-strapped schools and communities to implement fully and with fidelity. Evidence-based interventions are unlikely to make a large impact on their intended outcomes if they are too costly or cumbersome to scale up and implement widely (Wandersman et al. 2008). With this interest in mind, CDC is attempting to facilitate the implementation of the Dating Matters comprehensive model with the Dating Matters Toolkit, an online resource for communities where they can learn about Dating Matters and its various components, understand the resources needed to carry out the model as designed, access all CDC-developed components of the intervention as well as comprehensive implementation guidance for the entire model, complete online program facilitator training, and join a community of practice to collaborate with and learn from other communities implementing Dating Matters (Centers for Disease Control and Prevention 2019). The Toolkit was released in September 2019. CDC is actively engaged with some communities and organizations using the Toolkit to implement Dating Matters, providing opportunities to learn from their 'real-world' implementation and continue to improve and expand the Toolkit to meet their needs. In the first year since the release of the Toolkit, nearly 150 communities have requested access to the program materials and training indicating an interest in implementation. Forthcoming papers by CDC (expected 2021) will describe the development of the Toolkit to support the model's potential for widespread dissemination and estimate the cost of Dating Matters implementation to communities (see https:// www.cdc.gov/violenceprevention/intimatepartnerviolence/ datingmatters/pub-resources.html for an updated list of all Dating Matters-related publications). 
In conclusion, this multi-site RCT of the Dating Matters comprehensive prevention model found significant reductions in TDV relative to the standard of care Safe Dates model (Niolon et al. 2019). Together, the papers in this special section also demonstrate significant impacts on a host of other adolescent risk behaviors and experiences, including bullying and peer violence, sexual violence and sexual harassment, and weapon carrying, substance use, and other delinquent behaviors. These findings are consistent with the field's demand for interventions that can reduce multiple forms of interpersonal violence and other negative outcomes. By addressing shared risk and protective factors for these multiple outcomes (Centers for Disease Control and Prevention 2016; Niolon et al. in press), the Dating Matters model demonstrates promise as such a preventive intervention.

Funding N/A for this introduction.

\section{Compliance with Ethical Standards}

Conflict of Interest The author of this is introduction is employed by the $\mathrm{CDC}$, which developed the Dating Matters program. The author is also the Principle Investigator of the randomized controlled trial that evaluated the Dating Matters comprehensive prevention program, and an author on the three outcome papers included in this special section.

Ethics Approval N/A for this introduction.

Disclaimer The findings and conclusions in this introduction are those of the author and do not necessarily represent the official position of the Centers for Disease Control and Prevention.

Consent to Participate N/A for this introduction.

\section{References}

Basile, K. C., Clayton, H. B., DeGue, S., Gilford, J. W., Vagi, K. J., Suarez, N. A., Zwald, M. L., \& Lowry, R. (2020). Interpersonal Violence Victimization Among High School Students - Youth Risk Behavior Survey, United States, 2019. In: Youth risk behavior surveillance - United States, 2019. Morbidity and Mortality Weekly Report, 202069(Suppl 1), 28-37.

Centers for Disease Control and Prevention. (2016). Preventing multiple forms of violence: A strategic vision for connecting the dots. Atlanta: Division of Violence Prevention, National Center for Injury Prevention and Control, Centers for Disease Control and Prevention.

Centers for Disease Control and Prevention. (2019). The dating matters toolkit. Atlanta: National Center for Injury Prevention and Control, Centers for Disease and Prevention Available at: https:// vetoviolence.cdc.gov/apps/dating-matters-toolkit/.

Centers for Disease Control and Prevention (2020). Preventing Teen Dating Violence webpage. Available: https:/www.cdc.gov/ violenceprevention/intimatepartnerviolence/teendatingviolence/ fastfact.html. Accessed 08/31/2020.

David-Ferdon, C., \& Simon, T. R. (2014). Preventing Youth Violence: Opportunities for Action. Atlanta: National Center for Injury Prevention and Control, Centers for Disease Control and Prevention.
Debnam, K.J., \& Temple, J. R. (2020). Dating matters and the future of teen dating violence prevention. Prevention Science. https://doi.org/ 10.1007/s11121-020-01169-5.

Eaton, D. K., Kann, L., Kinchen, S., Shanklin, S., Ross, J., Hawkins, J., et al. (2010). Youth risk behavior surveillance-United States, 2009. MMWR Surveillance Summaries, 59(5), 1-142.

Exner-Cortens, D., Eckenrode, J., \& Rothman, E. (2013). Longitudinal associations between teen dating violence victimization and adverse health outcomes. Pediatrics, 131(1), 71-78.

Exner-Cortens, D., Eckenrode, J., Bunge, J., \& Rothman, E. (2017). Revictimization after adolescent dating violence in a matched, national sample of youth. Journal of Adolescent Health, 60(2), 176183.

Forehand, R., Armistead, L., Long, N., Wyckoff, S. C., Kotchick, B. A., Whitaker, D., et al. (2007). Efficacy of a parent-based sexual-risk prevention program for African American preadolescents: A randomized controlled trial. Archives of Pediatrics \& Adolescent Medicine, 161(12), 1123-1129. https://doi.org/10.1001/archpedi. 161.12.1123.

Foshee, V. A., Bauman, K. E., Ennett, S. T., Linder, G. F., Benefield, T., \& Suchindran, C. (2004). Assessing the long-term effects of the safe dates program and a booster in preventing and reducing adolescent dating violence victimization and perpetration. American Journal of Public Health, 94(4), 619-624. https://doi.org/10.2105/AJPH.94.4.619.

Foshee, V. A., Reyes, H. L. M., Ennett, S. T., Cance, J. D., Bauman, K. E., \& Bowling, J. M. (2012). Assessing the effects of families for safe dates, a family-based teen dating abuse prevention program. Journal of Adolescent Health, 51(4), 349-356. https://doi.org/10. 1016/j.jadohealth.2011.12.029.

Niolon, P. H., Taylor, B., Latzman, N. E., Vivolo-Kantor, A., Valle, L. A., \& Tharp, A. T. (2016). Lessons learned in evaluating a multi-site, comprehensive teen dating violence prevention strategy: Design and challenges of the evaluation of Dating Matters ${ }^{\circledR}$ : Strategies to Promote Healthy Teen Relationships. Psychology of Violence, 6(3), 452-458.

Niolon, P. H., Vivolo-Kantor, A. M., Tracy, A., Latzman, N. E., Little, T. D., DeGue, S., et al. (2019). An RCT of dating matters: effects on teen dating violence and relationship behaviors. American Journal of Preventive Medicine, 57(1), 13-23. https://doi.org/10.1016/j. amepre.2019.02.022.

Niolon, P.H., Treves-Kagan, S., Dahlberg, L.L., \& Mercy, J.A. (in press). The evolution of interpersonal violence research and prevention across the lifespan in the U.S.: The past, present and future. Invited chapter for Robert Geffner, victor Vieth, Viola Vaughan-Eden, Alan Rosenbaum, L. Kevin Hamberger and Jaquelyn white (Eds) book, Handbook of Interpersonal Violence Across the Lifespan.

Reed, E., Silverman, J. G., Raj, A., Decker, M. R., \& Miller, E. (2011). Male perpetration of teen dating violence: associations with neighborhood violence involvement, gender attitudes, and perceived peer and neighborhood norms. Journal of Urban Health, 88(2), 226-239.

Tharp, A. T., Burton, T., Friere, K., Hall, D. M., Harrier, S., Latzman, N. E., Luo, F., Niolon, P. H., Ramirez, M., \& Vagi, K. (2011). Dating matters: strategies to promote healthy teen relationships. Journal of Women's Health, 20, 1761-1765.

Walton, M. A., Cunningham, R. M., Goldstein, A. L., Chermack, S. T., Zimmerman, M. A., Bingham, C. R., et al. (2009). Rates and correlates of violent behaviors among adolescents treated in an urban emergency department. Journal of Adolescent Health, 45(1), 77-83.

Wandersman, A., Duffy, J., Flaspohler, P., Noonan, R., Lubell, K., Stillman, L., Blachman, M., Dunville, R., \& Saul, J. (2008). Bridging the gap between prevention research and practice: the interactive systems framework for dissemination and implementation. American Journal of Community Psychology, 41, 171-181.

Publisher's Note Springer Nature remains neutral with regard to jurisdictional claims in published maps and institutional affiliations. 